\title{
ALTERNATIVE ANALYSIS OF FINITE-TIME PROBABILITY DISTRIBUTIONS OF RENEWAL THEORY
}

\author{
PeRCY H. BRILL \\ Department of Mathematics and Statistics and Department of Management Science \\ University of Windsor, Canada \\ E-mail: brill@uwindsor.ca
}

\begin{abstract}
We introduce a level-crossing analysis of the finite time- $t$ probability distributions of the excess life, age, total life, and related quantities of renewal processes. The technique embeds the renewal process as one cycle of a regenerative process with a barrier at level $t$, whose limiting probability density function leads directly to the time- $t$ quantities. The new method connects the analysis of renewal processes with the analysis of a large class of stochastic models of Operations Research. Examples are given.
\end{abstract}

\section{INTRODUCTION}

The probability distributions of excess life, age, and total life at an arbitrary finite time $t$, and related quantities, are useful for analyzing renewal-theoretic models. They are derived typically via renewal integral equations obtained using renewal arguments, such as conditioning on the time of the first renewal. The time- $t$ probability distributions have been discussed by many authors including Feller [1-3], Doob [4], Smith [5], Cox [6], Ross [7], and Karlin and Taylor [8].

The conventional method of deriving and solving renewal integral equations for the finite time- $t$ distributions may encounter difficulties when attempting to solve the equations. Bartholemew [9], Deligonul [10], among others, address the need for using approximate solutions of the integral equations. Deligonul [10] mentions authors who have used: Laplace transforms, power series expansion, direct numerical solution of an approximate integral equation, and spline functions.

Other difficulties may arise when using the conventional method, especially if the interarrival times have finite support. Suppose the support is $(0, U)$, where $U \in(0, \infty)$ or $U=\infty$. If $t<U$, the difficulties of solving the renewal integral equation are similar to those discussed in Bartholemew [9] and Deligonul [10]. However, if $t>U$ (implying $U$ is finite) solving the renewal equation becomes increasingly difficult as $t$ increases because: (a) the excess life distribution must first be known at $n U, n=1, \ldots,\lfloor t / U\rfloor$, where $\lfloor\alpha\rfloor=$ (greatest integer $\leq \alpha$ ); (b) the calculation may require the $n$-fold self convolutions of the cdf (cumulative distribution function) of the inter-arrival time $(n=2,3, \ldots)$, which become more complex to compute as $n$ increases. The alternative analysis presented here is especially useful in obtaining the finite time- $t$ distributions and related quantities if $t>U$ (Section 5.2). 


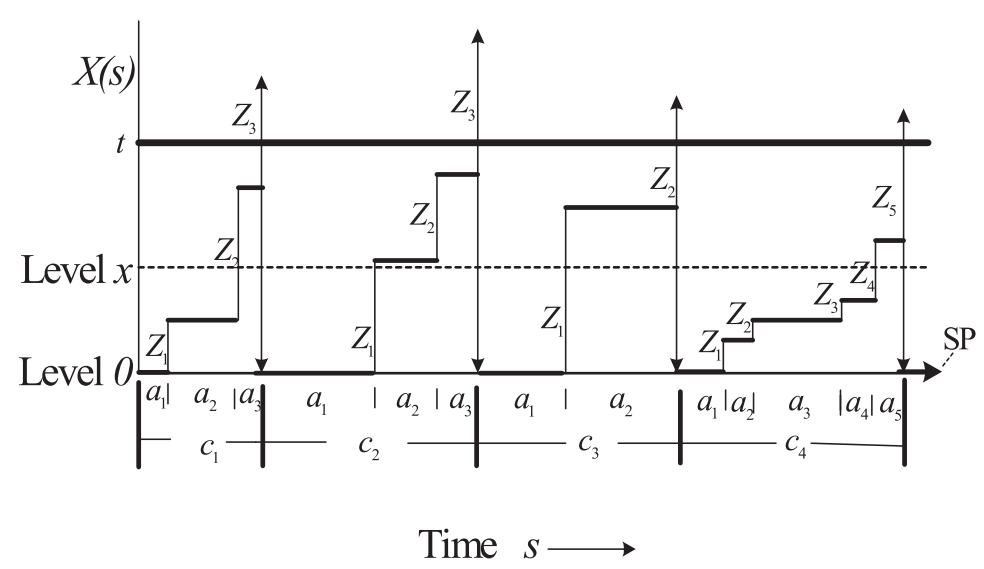

Figure 1. Sample path of regenerative process $\{X(s)\}_{s \geq 0}$ and renewal process $\left\{Z_{n}\right\}_{n=1,2, \ldots}$. SP denotes "System Point", the leading point of the sample path as it evolves over time. The $Z_{j}$ 's ( $j$ fixed) in different cycles are equal in distribution, but have different values.

The present paper considers an ordinary renewal process $\left\{Z_{n}\right\}_{n=1,2, \ldots}$ with independent identically distributed (i.i.d.) continuous inter-arrival times; let $Z_{n} \underset{\text { dist }}{\bar{Z}} Z$. In the new method, we transform the one-dimensional renewal process $\left\{Z_{n}\right\}_{n=1,2, \ldots}$ into a twodimensional positive recurrent regenerative process $\{X(s)\}_{s \geq 0}$ having state space $[0, \infty)$ with jumps $\underset{\text { dist }}{=} Z$ occurring at an arbitrary Poisson rate, and regenerative epochs at instants of level- $t$ exceedances (Section 2). The limiting pdf of $\{X(s)\}_{s \geq 0}$ exists (e.g., Sigman and Wolff [11]; Asmussen [12], Tijms [13]), and is concentrated on the state-space interval $[0, t)$. The limiting probability density function (pdf) is of fundamental importance because it leads directly to the finite time- $t$ distributions and related quantities. In this paper, we obtain the limiting pdf of $\{X(s)\}_{s}>0$ via a level-crossing procedure (Section 3.1). The idea of using a regenerative process $\{X(s)\}_{s}>0$ is based on the generic framework proposed in Brill [14]. The new analysis also applies to modified renewal processes by a slight modification (Section 5.3).

The new method has useful advantages. It bypasses ad-hoc renewal arguments in various cases, such as conditioning on the first renewal, etc., by focusing on the sample path of $\{X(s)\}_{s \geq 0}$. This makes the analysis straightforward, and faster and easier than the conventional method in many cases, because a sample path is a concrete guide to derive the limiting pdf of $\{X(s)\}_{s \geq 0}$ (e.g., Figure 1). Level-crossing rates are equal to simple mathematical expressions, or simple integral transforms, of the limiting pdf. Intuitive conservation laws connect the level-crossing rates and the integral equations for the limiting pdf (Section 3.1.1). Analytical properties of the pdf's of the time- $t$ quantities, e.g., discontinuities or general functional forms, can be readily determined using the new method. The existence of the limiting pdf of $\{X(s)\}_{s>0}$ implies that the time- $t$ distributions exist when $Z$ has no mean (e.g., a standard type-1 Pareto distribution with shape parameter less than or equal to 1). Level-crossing methods also lead to the time- $t$ quantities (Section 3.1.2). The new method connects the analysis of the time- $t$ quantities with the analyses of queues having bounded wait, inventories, dams, replacement models, and actuarial ruin models (Brill and $\mathrm{Yu}[15]$ ). It provides a completely different perspective of the time- $t$ quantities and suggests new avenues of research. References for level-crossing methods are Brill and 
Posner [16,17], Cohen [18,19], Brill [20,21] and others. Regenerative processes are discussed in Smith [5], Cohen [18], Sigman and Wolff [11], Asmussen [12], Tijms [13], and others.

Sections 2-4 consider the ordinary renewal process. Section 2 details the underlying regenerative process $\{X(s)\}_{s \geq 0}$. Section 3 describes the level-crossing procedure to be used in deriving the pdf's of interest; and establishes the connection between $\{X(s)\}_{s \geq 0}$ and the renewal function of $\left\{Z_{n}\right\}_{n=1,2, \ldots}$. Section 4 derives the time- $t$ distributions of the excess life, age, and total life. Section 5 gives examples, including a modified renewal process. Section 6 briefly compares the present work with the conventional method of renewal arguments and integral equations, and summarizes some contributions of the present paper.

\section{THE ORDINARY RENEWAL PROCESS AND REGENERATIVE PROCESS $\{X(s)\}_{s \geq 0}$}

Consider the renewal process $\left\{Z_{n}\right\}_{n=1,2, \ldots}$, where $Z_{n}$ is a continuous random variable on $(0, U)$ and $U \in(0, \infty)$ or $U=\infty$. Let $Z_{1} \underset{\text { dist }}{\equiv} Z_{n} \underset{\text { dist }}{\equiv} Z$ with the common cdf $B(\cdot), \operatorname{pdf} b(\cdot)$ and ccdf (complementary cumulative distribution function) $\bar{B}(\cdot)=1-B(\cdot)$. Let $B^{* n}(\cdot)$ denote the $n$-fold self convolution of $B(\cdot)$ and $b^{* n}(x)=(d / d x) B^{* n}(x), x>0$.

Fix arbitrary finite time $t>0$. Denote the time- $t$ excess life by $\gamma_{t}$, age by $\delta_{t}$ and total life by $\beta_{t}$, with cdf's $F_{\gamma_{t}}, F_{\delta_{t}}, F_{\beta_{t}}$ and pdf's $f_{\gamma_{t}}, f_{\delta_{t}}, f_{\beta_{t}}$, respectively. Denote the time- $t$ renewal function as $M(t)$. Then (e.g., Ross [22], Pinsky and Karlin [23])

$$
M(t)=\sum_{n=1}^{\infty} B^{* n}(t) ; \quad M^{\prime}(t)=\sum_{n=1}^{\infty} b^{* n}(t) .
$$

\subsection{Regenerative process}

Consider $\{X(s)\}_{s \geq 0}$ having state space $[0, \infty)$, constructed from $\left\{Z_{n}\right\}_{n=1,2, \ldots}$ and a Poisson process of arbitrary rate $\lambda$ ( $\equiv 1$ for simplicity) as follows. Let $X(0)=0$. Assume sample paths of $\{X(s)\}_{s \geq 0}$ are right continuous and have left limits. Let $\{X(s)\}_{s \geq 0}$ make upward jumps $\underset{\text { dist }}{=} Z$ at Poisson rate 1 , with inter-jump times $a_{i} \underset{\text { dist }}{=} a$ and $E(a) \equiv 1$. The jumps originate in $[0, t)$ and are in one-to-one correspondence with the $\left\{a_{i}\right\}_{i=1,2, \ldots}$ (Figure 1). Suppose $\sigma^{-}$is an instant just before an upward-jump of $\{X(s)\}_{s \geq 0}$, i.e., $X\left(\sigma^{-}\right)=\sum_{n=1}^{i} Z_{n}<t$ for some non-negative integer $i ;\left(\sum_{n=1}^{0}=0\right)$. Then just after the jump

$$
X(\sigma)= \begin{cases}X\left(\sigma^{-}\right)+Z & \text { if } X\left(\sigma^{-}\right)+Z<t, \\ 0 & \text { if } X\left(\sigma^{-}\right)+Z>t .\end{cases}
$$

If $X(\sigma)<t$, then $X(s) \equiv X(\sigma), s \in[\sigma, \sigma+a)$. If $X\left(\sigma^{-}\right)+Z>t$ then $X(s)=0$, $s \in[\sigma, \sigma+a)$. That is, whenever $X(s)$ overshoots level $t$ the renewal process $\left\{Z_{n}\right\}_{n=1,2}$ restarts at level 0 , and the Poisson process $\left\{a_{n}\right\}_{n=1,2, \ldots}$ continues at rate 1 . This dynamic creates a sequence of i.i.d. cycles between successive upcrossings of level $t$, in which the sample path is a random non-decreasing step function in $[0, t)$. At any instant in a cycle $\{X(s)\}_{s \geq 0}$ is equal to the time of the last renewal in $\left\{Z_{n}\right\}_{n=1,2, \ldots}$ (Figure 1 ).

The total probability of $\{X(s)\}_{s \geq 0}$ is supported on state-space interval $[0, t)$ because jumps $\underset{\text { dist }}{=} Z$ that start at some level $y \in[0, t)$ and upcross $t$ signal double jumps that upcross and downcross every level in $[y, y+Z)$. Thus, $\{X(s)\}_{s \geq 0}$ spends zero time above level $t$.

Since $\{X(s)\}_{s \geq 0}$ is a regenerative process, it has a limiting mixed pdf denoted by $\left\{\pi^{(t)}, f^{(t)}(x)\right\}_{0<x<t}($ e.g., Sigman and Wolff [11], Asmussen [12], Tijms [13]). Notably, the 
new method utilizes $\left\{\pi^{(t)}, f^{(t)}(x)\right\}_{0<x<t}$ to derive the time- $t$ distributions of $\gamma_{t}, \delta_{t}, \beta_{t}$, and related quantities (Section 4 ).

Remark 2.1: The length of every cycle of $\{X(s)\}_{s \geq 0}$ includes at least one interval $\underset{\text { dist }}{=} a$. Note that $P$ (jump upcrosses level $t \mid$ jump starts at level 0$)=\bar{B}(t)$. If $t<U$, then $\bar{B}(t)>0$, implying such a jump ends a cycle of length $\underset{\text { dist }}{=} a$; a new cycle starts immediately at level 0 . Thus, $\bar{B}(t)$ is the long run proportion of cycles having length $\underset{\text { dist }}{=} a$. If $t \geq U$, then $\bar{B}(t)=0$. Thus $P$ (jump upcrosses level $t$ jump starts in state-space interval $[0, t-U])=0$. However, $P$ (jump upcrosses level $t \mid$ jump starts in state-space interval $(t-U, t))>0$. Then a cycle has length $\underset{\text { dist }}{=} \sum_{n=2}^{i} a_{i}$, where $i \geq 2$ is finite.

Remark 2.2: $\{X(s)\}_{s \geq 0}$ is a Markov process on each regenerative cycle.

\section{PRELIMINARIES}

\subsection{Notes on the Level-Crossing Procedure Used in the Analysis}

This subsection introduces some ideas of the level-crossing procedure to be used in the following development. For exposition, here we assume that $t \leq U$. Cases where $t>U$ are detailed later where applicable. More level-crossing particulars are given in the references cited in Section 1.

3.1.1. Deriving the limiting pdf $\left\{\pi^{(t)}, f^{(t)}(x)\right\}_{0<x<t}$. Consider a sample path of $\{X(s)\}_{s \geq 0}$ (Figure 1$)$. Let $\mathcal{D}_{s}^{j}(x), x \in[0, \infty)$, denote the total number of jump downcrossings of level $x$ during $(0, s)$. Let $\mathcal{U}_{s}^{j}(x), x \in(0, \infty]$, denote the total number of jump upcrossings of level $x$ during $(0, s)$. (A downcrossing is a transition from state-space subset $(x, \infty)$ into $[0, x]$; an upcrossing is a transition from state-space subset $[0, x]$ into $(x, \infty)$.) For $\{X(s)\}_{s \geq 0}$ all level crossings are due to jumps. Conservation of sample-path upcrossing and downcrossing rates across level $x$, implies

$$
\left.\begin{array}{rl}
\lim _{s \rightarrow \infty} \frac{\mathcal{D}_{s}^{j}(x)}{s} & =\lim _{s \rightarrow \infty} \frac{\mathcal{U}_{s}^{j}(x)}{s}, x \in[0, \infty), a . s ., \\
\lim _{s \rightarrow \infty} \frac{E\left(\mathcal{D}_{s}^{j}(x)\right)}{s} & =\lim _{s \rightarrow \infty} \frac{E\left(\mathcal{U}_{s}^{j}(x)\right)}{s}, x \in[0, \infty) .
\end{array}\right\}
$$

All jumps that start in the state-space interval $[0, t)$ are upward jumps. Upward jumps that exceed level $t$ signal the ends of cycles, followed immediately by downward jumps that end at level 0 . Each downward jump downcrosses every $x \in[0, t)$. Our analysis includes entire jumps, not just the net jumps. When a jump upcrosses $t$ a double jump occurs. The first is the jump overshooting $t$; the second starts immediately at the end of the first jump, and is downward ending at level 0 . A net count of 0 crossings is given to every level in the state-space intersection of the double-jump pairs. If an upward jump starts at level 0 and exceeds level $t$ then the paired downward jump cancels the count of the upward jump at all $x \in(0, t)$, and the net total count of both jumps is 0 (see Remark 2.1).

Connection to mixed pdf $\left\{\pi^{(t)}, f^{(t)}(x)\right\}_{0<x<t}$. The mixed pdf $\left\{\pi^{(t)}, f^{(t)}(x)\right\}_{0<x<t}$ is a timeaverage pdf. Since jumps occur at a Poisson rate, the arrival-point pdf is the same as the 
time-average pdf (e.g., Wolff [24]). Thus

$$
\begin{gathered}
\lim _{s \rightarrow \infty} \frac{\mathcal{D}_{s}^{j}(x)}{s}=\lim _{s \rightarrow \infty} \frac{\mathcal{U}_{s}^{j}(t)}{s}=\lim _{s \rightarrow \infty} \frac{\mathcal{D}_{s}^{j}(0)}{s}=\pi^{(t)}, x \in[0, t), \\
\text { and } \lim _{s \rightarrow \infty} \frac{\mathcal{U}_{s}^{j}(x)}{s}=\pi^{(t)} \bar{B}(x)+\int_{0}^{x} \bar{B}(x-y) f^{(t)}(y) d y, x \in[0, t) .
\end{gathered}
$$

Explanation of equation (3.2). (downcrossing rate of $x)=($ upcrossing rate of $t$ ) because of the double jumps whenever $\{X(s)\}_{s \geq 0}$ exceeds $t$; (upcrossing rate of $\left.t\right)=$ (downcrossing rate of 0$)=($ entrance rate of $\{0\})=($ exit rate of $\{0\})=1 \cdot \pi^{(t)}$

Explanation of equation (3.3). $\lim _{s \rightarrow \infty}\left(\mathcal{U}_{s}^{j}(x) / s\right)=$ (rate of ju mps that start at level 0 and upcross $x)+$ (rate of jumps that start at levels $y \in(0, x)$ and upcross $x)$. The first term is $1 \cdot \pi^{(t)} \bar{B}(x)$, because the rate of jumps that start at level 0 is $1 \cdot \pi^{(t)}$, and $P($ jump exceeds $x \mid$ jump starts at level 0$)=\bar{B}(x)$. The second term is $1 \cdot \int_{0}^{x} \bar{B}(x-y) f^{(t)}(y) d y$, because the rate of jumps that start in state-space interval $(y, y+d y)$ is $1 \cdot f^{(t)}\left(y^{\prime}\right) d y$, for some $y^{\prime} \in(y, y+d y)$, and $P($ jump exceeds $x \mid$ jump starts at level $y \in(0, x))=\bar{B}(x-y)$. Thus, $\int_{0}^{x} \bar{B}(x-y) f^{(t)}(y) d y$ is the total rate at which $\{X(s)\}_{s \geq 0}$ upcrosses $x$ from starts at levels $y \in(0, x)$.

By (3.1), the expressions (3.2) and (3.3) are equal, resulting in the integral equation (3.5) below.

3.1.2. Deriving the $p d f f_{\gamma_{t}}(x), x>0$ : Consider a fixed level $(t+x), x>0$. We obtain two different expressions for the upcrossing rate of level $(t+x)$ : expression (i) in terms of $F_{\gamma_{t}}(x), x>0$; expression (ii) in terms of $\left\{\pi^{(t)}, f^{(t)}(x)\right\}_{0<x<t}$. Then we equate expressions (i) and (ii) to form an equation. If $\left\{\pi^{(t)}, f^{(t)}(x)\right\}_{0<x<t}$ is known (Section 3.1.1) then we can derive $F_{\gamma_{t}}(x), x>0$ or $f_{\gamma_{t}}(x), x>0$.

Expression (i) is $\pi^{(t)}\left(1-F_{\gamma_{t}}(x)\right)$, because the upcrossing rate of level $t$ is $1 \cdot \pi^{(t)}$ and $P($ jump upcrosses level $(t+x) \mid$ jump upcrosses level $t)=1-F_{\gamma_{t}}(x), 0<x<U$ (see Section 3.1.1).

Let $(\alpha, \beta)^{+}=\max (\alpha, \beta)$. Expression $(i i)$ is

$$
\pi^{(t)} \bar{B}(t+x)+\int_{y=(t+x-U, 0)^{+}}^{t} \bar{B}(t+x-y) f^{(t)}(y) d y, 0<x<U .
$$

In (3.4), the first term is the rate of jumps that start at level 0 and upcross $(t+x)$, because the rate of jumps that start from level 0 is $1 \cdot \pi^{(t)}$ and $P($ jump upcrosses level $(t+x) \mid$ jump starts at level 0$)=\bar{B}(t+x)$. The second term is the rate of jumps that start at levels $y \in\left((t+x-U, 0)^{+}, t\right)$ and upcross $(t+x)$, because $P($ jump upcrosses level $(t+$ $x) \mid$ jump starts at level $y)=\bar{B}(t+x-y)$. In (3.4), if $(t+x-U) \geq 0$ then $\bar{B}(t+x)=0$, and the lower limit of the integral is $(t+x-U)$. If $(t+x-U)<0$ then $\bar{B}(t+x)>0$, and the lower limit of the integral is 0 . Equating expressions $(i)$ and (ii) yields equation (4.1) below.

\subsection{Solution for $\left\{\pi^{(t)}, f^{(t)}(x)\right\}_{0<x<t}$}

We treat the cases $t \leq \boldsymbol{U}$ and $t>\boldsymbol{U}$ separately. 
3.2.1. Case $t \leq \boldsymbol{U}$ : For each level $x \in(0, t)$, we balance upcrossing and downcrossing rates as explained in Section 3.1.1, which yields the integral equation for $f^{(t)}(\cdot)$ (see Figure 1)

$$
\pi^{(t)} \bar{B}(x)+\int_{y=0}^{x} \bar{B}(x-y) f^{(t)}(y) d y=\pi^{(t)}, \quad x \in(0, t) .
$$

The normalizing condition is

$$
\pi^{(t)}+\int_{x=0}^{t} f^{(t)}(x) d x=1 .
$$

Dividing (3.5) by $\pi^{(t)}$ gives the identity

$$
\bar{B}(x)+\int_{y=0}^{x} \bar{B}(x-y) \frac{f^{(t)}(y)}{\pi^{(t)}} d y \equiv 1, \quad x \in(0, t) .
$$

Letting $x \uparrow t$ in (3.7) yields the useful formula

$$
\bar{B}(t)+\int_{y=0}^{t} \bar{B}(t-y) \frac{f^{(t)}(y)}{\pi^{(t)}} d y=1 .
$$

We next solve (3.5) and (3.6) simultaneously.

3.2.2. Solution for $\left\{\pi^{(t)}, f^{(t)}(x)\right\}_{0<x<t}$ : Taking $d / d x$ on both sides of (3.5) yields the Volterra integral equation

$$
f^{(t)}(x)=\pi^{(t)} b(x)+\int_{y=0}^{x} b(x-y) f^{(t)}(y) d y, \quad 0<x<t,
$$

with unique solution

$$
f^{(t)}(x)=\pi^{(t)} \sum_{n=1}^{\infty} b^{* n}(x), \quad 0<x<t,
$$

$$
\text { where } b(x) \equiv b^{* 1}(x) \text {. }
$$

Formula (3.10) follows by substituting $f^{(t)}(y)$ from (3.9) recursively into the integral in (3.9).

From (2.1) and (3.10)

$$
\begin{aligned}
f^{(t)}(x) & =\pi^{(t)} \sum_{n=1}^{\infty} \frac{d}{d x} B^{* n}(x)=\pi^{(t)} \frac{d}{d x} \sum_{n=1}^{\infty} B^{* n}(x)=\pi^{(t)} M^{\prime}(x), 0<x<t, \\
\frac{f^{(t)}(x)}{\pi^{(t)}} & =M^{\prime}(x), \quad 0<x<t .
\end{aligned}
$$

To solve for $\pi^{(t)}$, substitute (3.11) into (3.6); $\int_{x=0}^{t} M^{\prime}(x) d x=M(t)-M(0)=M(t)$ implies

$$
\pi^{(t)}+\pi^{(t)} M(t)=1, \quad \pi^{(t)}=\frac{1}{1+M(t)} .
$$

From (3.12) and (3.13)

$$
f^{(t)}(x)=\frac{M^{\prime}(x)}{1+M(t)}, 0<x<t
$$




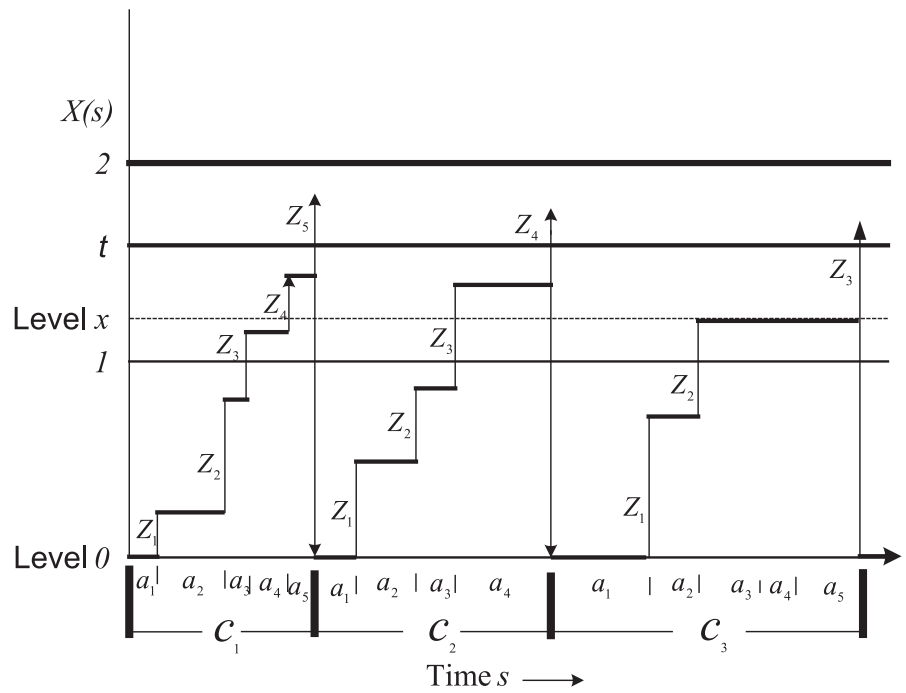

Figure 2. Sample path of regenerative process $\{X(s)\}_{s \geq 0}$ for $Z_{n} \underset{\text { dist }}{\equiv} U_{(0,1)}$ and $t \in(1,2)$. The $Z_{j}$ 's ( $j$ fixed) in different cycles are $\underset{\text { dist }}{=} U_{(0,1)}$, but have different values.

Expressing $M(x)(0<x \leq t)$ in terms of $\left\{\pi^{(t)}, f^{(t)}(y)\right\}_{0<y<x},(3.12)$ and (3.6) yield, respectively

$$
M(x)=\int_{y=0}^{x} M^{\prime}(y) d y=\frac{1}{\pi^{(t)}} \int_{y=0}^{x} f^{(t)}(y) d y, 0<x<t ; \quad M(t)=\frac{1}{\pi^{(t)}}-1 .
$$

Expected number of renewals required to first exceed $t$. Let $N_{t}=\min \left\{n \mid \sum_{i=1}^{n} Z_{i}>t\right\} . N_{t}$ is a stopping time for the sequence $\left\{Z_{i}\right\}_{i=1,2, \ldots}$ and also for the sequence $\left\{a_{i}\right\}_{i=1,2, \ldots .}$. Since $N_{t}=N(t)+1, E\left(N_{t}\right)=M(t)+1$. From $(3.15)$

$$
E\left(N_{t}\right)=\frac{1}{\pi^{(t)}} .
$$

Expected cycle length of $\{X(s)\}_{s \geq 0}$. Let $c$ denote a cycle length. Then $c=\sum_{i=1}^{N_{t}} a_{i}$. By Wald's equation and (3.16),

$$
E(c)=E\left(N_{t}\right) E(a)=E\left(N_{t}\right)=\frac{1}{\pi^{(t)}} .
$$

3.2.3. Case $t>U$ : Here $U<\infty$ since $t<\infty$ (see Figure 2). Suppose $t \in[N U,(N+$ 1) $U$ ) for some integer $N \geq 1$. Let $\boldsymbol{I}_{\boldsymbol{A}}(x)=1$ if $x \in \boldsymbol{A}$ and 0 otherwise.

Define $f_{n}^{(t)}(x)=f^{(t)}(x) \boldsymbol{I}_{[n U,(n+1) U)}(x), n=0,1, \ldots ;$ then

$$
f^{(t)}(x)=\sum_{n=0}^{N} f_{n}^{(t)}(x), 0<x<t .
$$

An integral equation for $f_{0}(x), 0<x<U$ is

$$
\pi^{(t)} \cdot \bar{B}(x)+\int_{0}^{x} \bar{B}(x-y) f_{0}^{(t)}(y) d y=\pi^{(t)}, 0<x<U
$$


which is the same as (3.5) except that the range's upper limit is $U$. Thus,

$$
f_{0}^{(t)}(x)=\pi^{(t)} M^{\prime}(x), 0<x<U,
$$

which is similar to (3.12). Integral equations for $f_{n}^{(t)}(x)$ in terms of $f_{n-1}^{(t)}(\cdot)$ are

$$
\begin{gathered}
\int_{y=x-U}^{n U} \bar{B}(x-y) f_{n-1}^{(t)}(y) d y+\int_{y=n U}^{x} \bar{B}(x-y) f_{n}^{(t)}(y) d y=\pi^{(t)}, \\
\text { where } \quad \begin{cases}n U \leq x<(n+1) U, & \text { if } n=0, \ldots, N-1 ; \\
N U \leq x<t, & \text { if } n=N .\end{cases}
\end{gathered}
$$

If $N=1$ then $U \leq t<2 U$, and (3.20) becomes

$$
\begin{gathered}
\int_{y=x-U}^{U} \bar{B}(x-y) f_{0}^{(t)}(y) d y+\int_{y=U}^{x} \bar{B}(x-y) f_{1}^{(t)}(y) d y=\pi^{(t)}, U \leq x<t, \\
\int_{y=x-U}^{U} \bar{B}(x-y) \pi^{(t)} M^{\prime}(y) d y+\int_{y=U}^{x} \bar{B}(x-y) f_{1}^{(t)}(y) d y=\pi^{(t)},
\end{gathered}
$$

which gives $f_{1}^{(t)}(\cdot)$ in terms of $f_{0}^{(t)}(\cdot)$. We solve (3.22) for $f_{1}^{(t)}(x), U \leq x<t$, in terms of $f_{0}(y), x-U<y<U$, using (3.20); or in terms of $\left\{\pi^{(t)}, M^{\prime}(y)\right\}_{x-U<y<U}$ using (3.22). Then solve recursively for $f_{n}^{(t)}(x), n=2,3, \ldots, N$, using (3.20). This gives $f^{(t)}(x)$ as a linear expression of functions of $x$ in which $\pi^{(t)}$ is a common factor. Then (3.6) yields $\pi^{(t)}$. (see example in Section 5.2.)

Remark 3.1: Formulas (3.15)-(3.17) for $M(t), E\left(N_{t}\right)$, and $E(c)$ apply when $t>U$.

\subsection{Limiting Proportion of Renewals of $\left\{Z_{n}\right\}_{n=1,2, \ldots}$ in $(r, v) \subseteq(0, t)$ in cycles of $\{X(s)\}_{s \geq 0}$}

3.3.1. Property of the limiting pdf of $\{X(s)\}_{s \geq 0}$ : Consider two different finite times $v$ and $t, 0<v<t$. In this subsection only, label the corresponding regenerative processes as $\left\{X^{(\xi)}(s)\right\}_{s \geq 0}, \xi=v, t$. We give a useful property connecting $f^{(v)}(x)$ and $f^{(t)}(x), 0<x<v$.

Observe that the integral equation for $f^{(v)}(x), 0<x<v$, is identical to (3.19) if $v \leq U$, or to (3.20) if $v>U$, with $\pi^{(t)}$ replaced by $\pi^{(v)}$. Hence, $f^{(v)}(x), 0<x<v$, is identical to $f^{(t)}(x), 0<x<v$, with $\pi^{(t)}$ replaced by $\pi^{(v)}$. Also, $\pi^{(v)}>\pi^{(t)}$ since the expected cycle length of $\left\{X^{(v)}(s)\right\}_{s \geq 0}$ is less than that of $\left\{X^{(t)}(s)\right\}_{s \geq 0}$. From Section 3.2.2 we obtain

$$
f^{(t)}(x)=\pi^{(t)} \cdot a(x), 0<x<v ; \quad f^{(v)}(x)=\pi^{(v)} \cdot a(x), 0<x<v,
$$

where $a(x)$ depends on $x$, but not on $v$ or $t$, i.e., $f^{(t)}(x)$ and $f^{(v)}(x)$ depend respectively on $t$ and $v$ through the factors $\pi^{(t)}$ and $\pi^{(v)}$ only. Since $\pi^{(v)}>\pi^{(t)}$ we have

$$
f^{(v)}(x)=\frac{\pi^{(v)}}{\pi^{(t)}} f^{(t)}(x)>f^{(t)}(x), 0<x<v .
$$

To illustrate (3.23) and (3.24), let $Z$ be uniform on $(0,1)$, and $t \leq 1$. Then (e.g., Brill [14])

$$
f^{(\xi)}(x)=\pi^{(\xi)} e^{x}, 0<x<\xi, \pi^{(\xi)}=e^{-\xi}, \text { for } \xi=v \text { and } \xi=t ; \quad \text { also } a(x)=e^{x} .
$$

Then $f^{(v)}(x)=\pi^{(v)} a(x)=\pi^{(v)}\left(f^{(t)}(x) / \pi^{(t)}\right)=e^{(t-v)} f^{(t)}(x)>f^{(t)}(x), 0<x<v$. 
3.3.2. Limiting proportion of renewals of $\left\{Z_{n}\right\}_{n=1,2, \ldots}$ in $(r, v) \subseteq(0, t)$ : Assume $f^{(t)}(x), 0<x<v$, and $f^{(v)}(x), 0<x<v$, are known (Section 3.2). Remark 3.1 and (3.24) imply

$$
M(v)=\frac{1}{\pi^{(v)}}-1=\frac{\left.\int_{y=0}^{v} f^{(v)} y\right) d y}{\pi^{(v)}}=\frac{\left.\int_{y=0}^{v} f^{(t)} y\right) d y}{\pi^{(t)}},
$$

which reflects the observation that $M(v)$ is invariant whether computed using $\left\{X^{(t)}(s)\right\}_{s \geq 0}$ or using $\left\{X^{(v)}(s)\right\}_{s \geq 0}$.

Consider a sample path of $\left\{X^{(t)}(s)\right\}_{s \geq 0}$ (Figure 1). Let $0<r<v<t$. Define $R_{(r, v)}(s)$ as the total number of renewals of $\left\{Z_{n}\right\}_{n=1,2, \ldots}$ in the state-space interval $(r, v), 0<r<v<t$, in the cycles of $\left\{X^{(t)}(s)\right\}_{s \geq 0}$ during $(0, s), s>0$. From the renewal reward theorem (e.g., Sigman and Wolff [11], Asmussen [12], Tijms [13]), and (3.17),

$$
\lim _{s \rightarrow \infty} \frac{R_{(r, v)}(s)}{s}=\frac{E(\text { number of renewals in }(r, v) \text { in a cycle })}{E(\text { cycle length })}=\frac{M(v)-M(r)}{\left(\frac{1}{\pi^{(t)}}\right)} .
$$

From (3.25) and (3.26)

$$
\left.\lim _{s \rightarrow \infty} \frac{R_{(r, v)}(s)}{s}=\frac{\left.\left.\int_{y=0}^{v} f^{(t)} y\right) d y-\int_{y=0}^{r} f^{(t)} y\right) d y}{\pi^{(t)}\left(\frac{1}{\pi^{(t)}}\right)}=\int_{y=r}^{v} f^{(t)} y\right) d y .
$$

Dividing and multiplying $\frac{R_{(r, v)}(s)}{s}$ by $R_{(0 . t)}(s)$ (which is positive for sufficiently large $s$ ) yields

$$
\left.\lim _{s \rightarrow \infty}\left(\frac{R_{(r, v)}(s)}{R_{(0, t)}(s)}\right) \cdot \lim _{s \rightarrow \infty}\left(\frac{R_{(0, t)}(s)}{s}\right)=\lim _{s \rightarrow \infty}\left(\frac{R_{(r, v)}(s)}{R_{(0 . t)}(s)}\right) \cdot \int_{0}^{t} f^{(t)}(y) d y=\int_{y=r}^{v} f^{(t)} y\right) d y .
$$

Therefore, the limiting proportion of renewals of $\left\{Z_{n}\right\}_{n=1,2, \ldots}$ in $(r, v) \subseteq(0, t)$ in cycles of $\{X(s)\}_{s \geq 0}$ as $s \rightarrow \infty$ is

$$
\lim _{s \rightarrow \infty}\left(\frac{R_{(r, v)}(s)}{R_{(0 . t)}(s)}\right)=\frac{\int_{r}^{v} f^{(t)}(y) d y}{\int_{0}^{t} f^{(t)}(y) d y}
$$

Intuitively, if we would observe, each up to time $t$, a sequence of statistically identical renewal processes $\left\{Z_{n}\right\}_{n=1,2, \ldots}$, then the proportion of renewals in $(r, v) \rightarrow(3.27)$. Alternatively, if we would observe up to time $t$, independent renewal processes, statistically identical to $\left\{Z_{n}\right\}_{n=1,2,,,}$, then the proportion of renewals in $(r, v) \rightarrow(3.27)$ as the number of observed processes $\rightarrow \infty$.

\section{DISTRIBUTIONS OF THE TIME- $t$ RANDOM VARIABLES IN TERMS OF $\left\{\pi^{(t)}, f^{(t)}(x)\right\}$}

We develop results separately for the cases $t \leq U$ and $t>U$. If $t \leq U$ then $U$ is finite or infinite. If $t>U$ then $U$ is necessarily finite, and we assume $t \in[N U,(N+1) U)$ for some integer $N \geq 1$. 


\subsection{Excess Life $\gamma_{t}$}

4.1.1. Case $t \leq \boldsymbol{U}$ : We obtain the following integral equation (4.1) for $\bar{F}_{\gamma_{t}}(x)$ by equating two different upcrossing rates of level $t+x$, as explained in Section 3.1.2.

$$
\begin{aligned}
\pi^{(t)} \bar{F}_{\gamma_{t}}(x) & =\pi^{(t)} \bar{B}(t+x)+\int_{y=(t+x-U, 0)^{+}}^{t} \bar{B}(t+x-y) f^{(t)}(y) d y, 0<x<U, \\
\bar{F}_{\gamma_{t}}(x) & =\bar{B}(t+x)+\int_{y=(t+x-U, 0)^{+}}^{t} \bar{B}(t+x-y) \frac{f^{(t)}(y)}{\pi^{(t)}} d y, 0<x<U .
\end{aligned}
$$

Taking $d / d x$ in (4.2) gives, since $\bar{B}(t+x-(t+x-U))=\bar{B}(U)=0$,

$$
f_{\gamma_{t}}(x)=b(t+x)+\int_{y=(t+x-U, 0)^{+}}^{t} b(t+x-y) \frac{f^{(t)}(y)}{\pi^{(t)}} d y, 0<x<U .
$$

From (4.3) we obtain

$$
f_{\gamma_{t}}(x)= \begin{cases}b(t+x)+\int_{y=0}^{t} b(t+x-y) \frac{f^{(t)}(y)}{\pi^{(t)}} d y, & 0<x<U-t, \\ \int_{y=t+x-U}^{t} b(t+x-y) \frac{f^{(t)}(y)}{\pi^{(t)}} d y, & U-t<x<U .\end{cases}
$$

Only (4.4a) holds if $U=\infty$. Both (4.4a) and (4.4b) hold if $U<\infty$.

Property If $t<U<\infty$ then $f_{\gamma_{t}}(x)$ has a jump discontinuity at $x=(U-t)$ of magnitude $f_{\gamma_{t}}\left((U-t)^{+}\right)-f_{\gamma_{t}}\left((U-t)^{-}\right)=-b\left(U^{-}\right)$. This follows by letting $x \downarrow(U-t)$ and $x \uparrow(U-t)$ in $(4.4 \mathbf{b})$ and $(4.4 \mathbf{a})$, respectively, and subtracting.

4.1.2. Case $t>\boldsymbol{U}:$ An equation analogous to (4.2) is

$$
\bar{F}_{\gamma_{t}}(x)=\int_{y=t+x-U}^{t} \bar{B}(t+x-y) \frac{f^{(t)}(y)}{\pi^{(t)}} d y, 0<x<U .
$$

Taking $d / d x$ in $(4.5)$, noting $\bar{B}(U)=0$, gives

$$
f_{\gamma_{t}}(x)=\int_{y=t+x-U}^{t} b(t+x-y) \frac{f^{(t)}(y)}{\pi^{(t)}} d y, \quad 0<x<U .
$$

From (4.6), with $\left\{f_{n}^{(t)}(y)\right\}_{n=0, \ldots, N}$ defined just before (3.18), we get

$$
f_{\gamma_{t}}(x)= \begin{cases}\int_{y=t+x-U}^{N U} b(t+x-y) \frac{f_{N-1}^{(t)}(y)}{\pi^{(t)}} d y \\ +\int_{y=N U}^{t} b(t+x-y) \frac{f_{N}^{(t)}(y)}{\pi^{(t)}} d y, \quad 0<x<(N+1) U-t, \\ \int_{y=t+x-U}^{t} b(t+x-y) \frac{f_{N}^{(t)}(y)}{\pi^{(t)}} d y, \quad(N+1) U-t<x<U .\end{cases}
$$

An example using (4.7) is given in Section 5.2. 


\subsection{Position Just Before $\{X(s)\}_{s \geq 0}$ Exceeds $t$}

We define $X_{J B}^{(t)}$ as the position of $\{X(s)\}_{s \geq 0}$ just before the jump that first exceeds level $t$. It is related to the age $\delta_{t}$. Denote its mixed pdf as $\left\{\pi_{J B}^{(t)}, f_{J B}^{(t)}(x)\right\}_{0<x<t}$ where $\pi_{J B}^{(t)}=$ $P\left(X_{J B}^{(t)}=0\right)$. (Note that $X_{J B}^{(t)}$ is an important quantity in various stochastic models, such as actuarial ruin models, e.g., Gerber and Shiu [25]).

4.2.1. Case $t \leq U$ : Consider a sample path of $\{X(s)\}_{s \geq 0}$ (Figure 1). Application of Baye's rule and using (3.8), yields

$f_{J B}^{(t)}(x) d x=\frac{P(\text { jump upcrosses } t \mid \text { jump starts at level } x>0) \cdot P(\text { jump starts at level } x) d x}{P(\text { a jump upcrosses } t)}$,

$$
f_{J B}^{(t)}(x)=\frac{\bar{B}(t-x) f^{(t)}(x)}{\pi^{(t)} \bar{B}(t)+\int_{y=0}^{t} \bar{B}(t-y) f^{(t)}(y) d y}=\bar{B}(t-x) \frac{f^{(t)}(x)}{\pi^{(t)}}, \quad 0<x<t .
$$

Also $\pi_{J B}^{(t)}=\frac{P(\text { jump upcrosses } t \mid \text { jump starts at level } 0) \cdot P(\text { jump starts at level } 0)}{P(\text { a jump upcrosses } t)}$,

$$
\pi_{J B}^{(t)}=\frac{\bar{B}(t) \pi^{(t)}}{\pi^{(t)} \bar{B}(t)+\int_{y=0}^{t} \bar{B}(t-y) f^{(t)}(y) d y}=\bar{B}(t) .
$$

Using (3.8) shows that $\left\{\pi_{J B}^{(t)}, f_{J B}^{(t)}(x)\right\}_{0<x<t}$ satisfies the law of total probability.

\subsubsection{Case $t>U$ :}

$$
f_{J B}^{(t)}(x)=\left\{\begin{array}{c}
\frac{\bar{B}(t-x) f_{N-1}^{(t)}(x)}{\int_{y=t-U}^{N U} \bar{B}(t-y) f_{N-1}^{(t)}(y) d y+\int_{y=N U}^{t} \bar{B}(t-y) f_{N}^{(t)}(y) d y} \\
=\frac{\bar{B}(t-x) f_{N-1}^{(t)}(x)}{\pi^{(t)}}, t-U \leq x<N U, \\
\frac{\bar{B}(t-x) f_{N}^{(t)}(x)}{\int_{y=t-U}^{N U} \bar{B}(t-y) f_{N-1}^{(t)}(y) d y+\int_{y=N U}^{t} \bar{B}(t-y) f_{N}^{(t)}(y) d y} \\
=\frac{\bar{B}(t-x) f_{N}^{(t)}(x)}{\pi^{(t)}}, N U \leq x<t .
\end{array}\right.
$$

\subsection{Age $\delta_{t}$}

We obtain the mixed pdf $\left\{\pi_{\delta_{t}}, f_{\delta_{t}}(x)\right\}_{0<x<t}$ using $\left\{\pi_{J B}^{(t)}, f_{J B}^{(t)}(x)\right\}_{0<x<t}$ of Section 4.2. Note that $\delta_{t}=t-X_{J B}^{(t)}, \pi_{\delta_{t}}=P\left(\delta_{t}=t\right)=P\left(X_{J B}^{(t)}=0\right)=\pi_{J B}^{(t)}$, and $f_{\delta_{t}}(x)=f_{J B}^{(t)}(t-x)$, $0<x<t$.

4.3.1. Case $\boldsymbol{t} \leq \boldsymbol{U}$ : Using (4.8) and (4.9) yields

$$
\pi_{\delta_{t}}=\bar{B}(t), \quad f_{\delta_{t}}(x)=\bar{B}(x) \frac{f^{(t)}(t-x)}{\pi^{(t)}}, \quad 0<x<t .
$$

By (3.8) $\left\{\pi_{\delta_{t}}, f_{\delta_{t}}(x)\right\}_{0<x<t}$, satisfies the law of total probability. 
4.3.2. Case $\boldsymbol{t}>\boldsymbol{U}$ : Note that $\pi_{\delta_{t}}=0$ since $t>U$. Using (4.10) and applying $f_{\delta_{t}}(x)=$ $f_{J B}^{(t)}(t-x)$, yields

$$
f_{\delta_{t}}(x)= \begin{cases}\frac{\bar{B}(x) f_{N}^{(t)}(t-x)}{\pi^{(t)}}, & 0<x<t-N U, \\ \frac{\bar{B}(x) f_{N-1}^{(t)}(t-x)}{\pi^{(t)}}, & t-N U<x<U .\end{cases}
$$

$\int_{0}^{U} f_{\delta_{t}}(x) d x=1$ follows by integration of $(4.12)$ on $(0, U)$.

\subsection{Total Life $\beta_{t}$}

The total life is $\beta_{t}=\gamma_{t}+\delta_{t}$. Hence, $P\left(\beta_{t}=x\right) d x=P\left(\gamma_{t}=x-\delta_{t}\right) d x, x>\delta_{t}$.

\subsubsection{Case $\boldsymbol{t} \leq \boldsymbol{U}$ :}

$$
f_{\beta_{t}}\left(x \mid \delta_{t}=y\right) d x=P(Z=x \mid Z>y) d x=\frac{P(Z=x) d x}{P(Z>y)}=\frac{b(x) d x}{\bar{B}(y)} .
$$

Unconditioning $f_{\beta_{t}}\left(x \mid \delta_{t}=y\right)$ with respect to $f_{\delta_{t}}(y)$, and substituting for $f_{\delta_{t}}(y)$ from (4.11), gives

$$
\begin{aligned}
f_{\beta_{t}}(x) & =\int_{y=0}^{x} \frac{b(x)}{\bar{B}(y)} f_{\delta_{t}}(y) d y \\
& =\int_{y=0}^{x} \frac{b(x)}{\bar{B}(y)} \bar{B}(y) \frac{f^{(t)}(t-y)}{\pi^{(t)}} d y=b(x) \int_{y=0}^{x} \frac{f^{(t)}(t-y)}{\pi^{(t)}} d y, \quad 0<x<t .
\end{aligned}
$$

Similar reasoning yields

$$
f_{\beta_{t}}(x)=\frac{b(x)}{\bar{B}(t)} \pi_{\delta_{t}}+b(x) \int_{y=0}^{t} \frac{f^{(t)}(t-y)}{\pi^{(t)}} d y=b(x)\left(1+\int_{y=0}^{t} \frac{f^{(t)}(t-y)}{\pi^{(t)}} d y\right), \quad t<x<U .
$$

Formulas (4.13) and (4.14) imply $f_{\beta_{t}}(x)$ has a jump discontinuity at $x=t$ of magnitude

$$
f_{\beta_{t}}\left(t^{+}\right)-f_{\beta_{t}}\left(t^{-}\right)=b(t) .
$$

If $U=\infty$ then $\lim _{t \rightarrow \infty} b(t)=0$, and the discontinuity in (4.15) vanishes as $t \rightarrow \infty$.

The law of total probability follows using integration by parts, $\bar{B}(U)=0$, and applying (3.8).

Check of $\lim _{t \rightarrow \infty} f_{\beta_{t}}(x)$. Formula (4.13) leads to the well-known formula $\lim _{t \rightarrow \infty} f_{\beta_{t}}(x)=$ $(x b(x)) / E(Z), x>0$. By $(3.12), f^{(t)}(t-y) / \pi^{(t)}=M^{\prime}(t-y)$. From (4.13)

$$
f_{\beta_{t}}(x)=b(x) \int_{y=0}^{x} M^{\prime}(t-y) d y=b(x)(M(t)-M(t-x)), 0<x<t .
$$

The basic renewal theorem (Blackwell's theorem, e.g., Karlin and Taylor [8], p. 191) implies

$$
\lim _{t \rightarrow \infty}(M(t)-M(t-x))=\frac{x}{E(Z)}, x>0 \Longrightarrow \lim _{t \rightarrow \infty} f_{\beta_{t}}(x)=\frac{x b(x)}{E(Z)}, x>0 .
$$


4.4.2. Case $\boldsymbol{t}>\boldsymbol{U}$ : Expressing $f_{\beta_{t}}(x)$ in terms of $f_{\beta_{t}}\left(x \mid \delta_{t}=y\right)$ and substituting for $f_{\delta_{t}}(y)$ from (4.12) yields

$$
f_{\beta_{t}}(x)=\int_{y=0}^{x} f_{\beta_{t}}\left(x \mid \delta_{t}=y\right) f_{\delta_{t}}(y) d y=b(x) \int_{y=0}^{x} \frac{f_{N}^{(t)}(t-y)}{\pi^{(t)}} d y, \quad 0<x<t-N U .
$$

Reasoning as for (4.16) and using also (4.12), yields

$$
f_{\beta_{t}}(x)=b(x)\left(\int_{y=0}^{t-N U} \frac{f_{N}^{(t)}(t-y)}{\pi^{(t)}} d y+\int_{y=t-N U}^{x} \frac{f_{N-1}^{(t)}(t-y)}{\pi^{(t)}} d y\right), \quad t-N U<x<U .
$$

\section{EXAMPLES}

\subsection{Generalized Hyper-Exponential (GH) Inter-Arrival Times}

The GH family is dense in the set of all distributions on $[0, \infty)$, and has coefficients of variation in $(0, \infty)$ (Botta et al. [26]). More properties are in Botta and Harris [27] and Harris et al. [28]. Heavy-tailed distributions, e.g., Pareto, etc., can be fitted by GH's (Yu et al. [29]).

Assume $Z_{n}, n=1,2, \ldots$ have $\operatorname{ccdf} \quad \bar{B}(x)=\sum_{i=1}^{N} a_{i} e^{-\mu_{i} x}, \quad x \geq 0, \quad \operatorname{pdf} \quad b(x)=$ $\sum_{i=1}^{N} a_{i} \mu_{i} e^{-\mu_{i} x}, x>0$, where $-\infty<a_{i}<\infty$, integer $N$ is positive, and $\sum_{i=1}^{N} a_{i}=1$. The hyperexponential is $\mathrm{GH}$ with $a_{i} \in(0,1), i=1, \ldots, N$. The exponential is hyperexponential when $N=1$.

$\left\{\boldsymbol{\pi}^{(t)}, \boldsymbol{f}^{(t)}(\boldsymbol{x})\right\} \quad$ Substituting $\bar{B}(x)$ into (3.5) and applying the differential operator $\prod_{i=1}^{N}\left\langle D+\mu_{i}\right\rangle$ leads to an $(N-1)^{\text {th }}$ order differential equation with constant coefficients for $f^{(t)}(x)$,

$$
\sum_{i=1}^{N} \prod_{j \neq i=1}^{N} a_{i}\left\langle D+\mu_{j}\right\rangle f^{(t)}(x)=\left(\prod_{i=1}^{N} \mu_{i}\right) \pi^{(t)}, 0<x<t
$$

We will illustrate the time- $t$ quantities for $N=2$. Solving (5.1) gives

$$
f^{(t)}(x)=\frac{\mu_{1} \mu_{2}}{A} \pi^{(t)}+C e^{-A x}, 0<x<t,
$$

where $A=a_{2} \mu_{1}+a_{1} \mu_{2}, C=H \pi^{(t)}$ and $H=\left(\mu_{1}-\left(\left(\mu_{1} \mu_{2}\right) / A\right)-a_{2}\left(\mu_{1}-\mu_{2}\right)\right)$. The normalizing condition (3.6) results in

$$
\pi^{(t)}=\frac{A}{A+\mu_{1} \mu_{2} t+H\left(1-e^{-A t}\right)}, \quad f^{(t)}(x)=\pi^{(t)}\left(\frac{\mu_{1} \mu_{2}}{A}+H e^{-A x}\right), 0<x<t .
$$

$\boldsymbol{f}_{\boldsymbol{\gamma}_{t}}(\boldsymbol{x})$ Before deriving $f_{\gamma_{t}}(\cdot)$ for $N=2$, we derive a general property for $f_{\gamma_{t}}(x), x>0$, $N^{t} \geq 2$. 
Property Define $a_{i}^{\prime} \equiv\left[e^{-\mu_{i} t}\left(1+\int_{y=0}^{t} e^{\mu_{i} y} \frac{f^{(t)}(y)}{\pi^{(t)}} d y\right)\right] \cdot a_{i}$. If $Z$ is GH with $\operatorname{ccdf} \bar{B}(x)=$ $\sum_{i=1}^{N} a_{i} e^{-\mu_{i} x}, x>0, N \geq 2$, then $\gamma_{t}$ is GH with

$$
\bar{F}_{\gamma_{t}}(x)=\sum_{i=1}^{N} a_{i}^{\prime} e^{-\mu_{i} x}, x>0, \quad f_{\gamma_{t}}(x)=\sum_{i=1}^{N} a_{i}^{\prime} \mu_{i} e^{-\mu_{i} x}, x>0 .
$$

This follows from (4.2), which gives

$$
\bar{F}_{\gamma_{t}}(x)=\sum_{i=1}^{N}\left[a_{i} e^{-\mu_{i} t}\left(1+\int_{y=0}^{t} e^{\mu_{i} y} \frac{f^{(t)}(y)}{\pi^{(t)}} d y\right)\right] e^{-\mu_{i} x}=\sum_{i=1}^{N} a_{i}^{\prime} e^{-\mu_{i} x}, x>0 .
$$

Also, $\sum_{i=1}^{N} a_{i}^{\prime}=1$ by comparison with (3.8).

Continuing with $N=2,(5.4)$ and (5.3) imply

$$
f_{\gamma_{t}}(x)=\sum_{i=1}^{2} a_{i} e^{-\mu_{i} t}\left(\mu_{i}+\frac{\mu_{1} \mu_{2}}{A}\left(e^{\mu_{i} t}-1\right)+\mu_{i} H \frac{e^{\left(\mu_{i}-A\right) t}-1}{\left(\mu_{i}-A\right)}\right) e^{-\mu_{i} x}, x>0
$$

$\left\{\boldsymbol{\pi}_{\boldsymbol{\delta}_{t}}, \boldsymbol{f}_{\delta_{t}}(\boldsymbol{x})\right\} \quad$ Applying (4.11), gives

$$
\pi_{\delta_{t}}=\sum_{i=1}^{2} a_{i} e^{-\mu_{i} t}, \quad f_{\delta_{t}}(x)=\left(\sum_{i=1}^{2} a_{i} e^{-\mu_{i} x}\right)\left(\frac{\mu_{1} \mu_{2}}{A}+H e^{-A(t-x)}\right), 0<x<t .
$$

$\boldsymbol{f}_{\beta_{t}}(\boldsymbol{x}) \quad$ Applying (4.13) and (4.14) gives

$$
f_{\beta_{t}}(x)=\left\{\begin{array}{l}
\left(\sum_{i=1}^{2} a_{i} \mu_{i} e^{-\mu_{i} x}\right)\left(\int_{y=0}^{x}\left(\frac{\mu_{1} \mu_{2}}{A}+H e^{-A(t-y)}\right) d y\right), x<t \\
\left(\sum_{i=1}^{2} a_{i} \mu_{i} e^{-\mu_{i} x}\right)\left(1+\int_{y=0}^{x}\left(\frac{\mu_{1} \mu_{2}}{A}+H e^{-A(t-y)}\right) d y\right), t<x<\infty
\end{array}\right.
$$

with a jump discontinuity at $x=t$ of magnitude $f_{\beta_{t}}\left(t^{+}\right)-f_{\beta_{t}}\left(t^{-}\right)=\left(\sum_{i=1}^{2} a_{i} \mu_{i} e^{-\mu_{i} t}\right)=$ $b(t)$.

Poisson Process as a special case of $G H$ inter-arrival times.

$\left\{\boldsymbol{\pi}^{(t)}, \boldsymbol{f}^{(t)}(\boldsymbol{x})\right\} \quad$ In $(5.3)$ if $\mu_{2}=\mu_{1}=\mu$ then $N=1, \mu_{1} \mu_{2}=\mu^{2}, A=\mu, H=0$, implying

$$
\pi^{(t)}=\frac{1}{1+\mu t}, \quad f^{(t)}(x)=\frac{\mu}{1+\mu t}, \quad 0<x<t .
$$

$f_{\gamma_{t}}(\boldsymbol{x}) \quad$ In (5.5) if $\mu_{i} \equiv \mu$ then $N=1,\left(\mu_{1} \mu_{2}\right) / A=\mu$, and $H=0$, implying $f_{\gamma_{t}}(x)=$ $\mu e^{-\mu x}, x>0$, which agrees with the memoryless property of the exponential. 
$\left\{\boldsymbol{\pi}_{\delta_{t}}, \boldsymbol{f}_{\delta_{t}}\right\} \quad$ From $(5.6)$

$$
\pi_{\delta_{t}}=e^{-\mu t}, \quad f_{\delta_{t}}(x)=e^{-\mu x} \frac{\left(\frac{\mu}{1+\mu t}\right)}{\left(\frac{1}{1+\mu t}\right)}=\mu e^{-\mu x}, 0<x<t .
$$

Recall $\pi_{\delta_{t}}=P\left(\delta_{t}=t\right)$. Formula (5.9) agrees with the well-known result (e.g., Karlin and Taylor, [8]). Note that $E\left(\delta_{t}\right)=1 / \mu-\left(e^{-\mu t}\right) / \mu$.

$\boldsymbol{f}_{\boldsymbol{\beta}_{t}}(\boldsymbol{x})$ Formula (5.7) yields

$$
f_{\beta_{t}}(x)=e^{-\mu x}(\mu x) \mu \boldsymbol{I}_{(\mathbf{0}, t)}(x)+\mu e^{-\mu x}(1+\mu t) \boldsymbol{I}_{(t, \infty)}(x) .
$$

with a discontinuity $f_{\beta_{t}}\left(t^{+}\right)-f_{\beta_{t}}\left(t^{-}\right)=\mu e^{-\mu t}$. Also $E\left(\beta_{t}\right)=(2 / \mu)-\left(e^{-\mu t} / \mu\right)$ and $\lim _{t \rightarrow \infty} E\left(\beta_{t}\right)=2 / \mu$.

Limiting proportion of renewals of $\left.\left\{Z_{n}\right\}_{n=1,2, \ldots} \in(\boldsymbol{r}, \boldsymbol{v}) \subseteq(\mathbf{0}, \boldsymbol{t})\right)$. Denote the limiting proportion of renewals of $\left\{Z_{n}\right\}_{n=1,2, \ldots}$ in $(r, v)$ during $(0, t)$ in the cycles of $\{X(s)\}_{s \geq 0}$ as $s \rightarrow \infty$ by

$$
p_{(r, v)}^{(t)}=\lim _{s \rightarrow \infty}\left(\frac{R_{(r, v)}(s)}{R_{(0 . t)}(s)}\right)
$$

From (3.27)

$$
p_{(r, v)}^{(t)}=\frac{(v-r)}{t}
$$

which is intuitive since the pdf $f^{(t)}(x)$ in $(5.8)$ is uniform on $(0, t)$.

\subsection{Uniformly Distributed Inter-Arrival Times}

Assume $\left\{Z_{n}\right\}$ are uniformly distributed on $(0,1)$ (here $U=1$ ). Then $b(y)=1,0<y<1$, and $\bar{B}(y)=(1-y), 0 \leq y<1$. We illustrate a case where $1<t<2$. (Note that $t>U$.)

Case Fixed $t \in(1,2]$. See Figure 2. Using the same notation for $f^{(t)}(x)$ and $f_{n}^{(t)}(x), n=$ 0,1 , as in formula (3.18), let

$$
f^{(t)}(x)=f_{0}^{(t)}(x)+f_{1}^{(t)}(x), 0<x<t, \quad \pi^{(t)}=\lim _{s \rightarrow \infty} P(X(s)=0) .
$$

$\boldsymbol{f}^{(t)}(\boldsymbol{x}) \quad$ Fix $t \in(1,2]$. From (3.19), (3.20), and (3.6) we get

$$
\begin{gathered}
\pi^{(t)} \cdot(1-x)+\int_{0}^{x}(1-x+y) f_{0}^{(t)}(y) d y=\pi^{(t)}, 0<x<1 \\
\int_{y=x-1}^{1}(1-x+y) f_{0}^{(t)}(y) d y+\int_{y=1}^{x}(1-x+y) f_{1}^{(t)}(y) d y=\pi^{(t)}, 1<x<t \\
\pi^{(t)}+\int_{0}^{1} f_{0}^{(t)}(y) d y+\int_{1}^{t} f_{1}^{(t)}(y) d y=1 .
\end{gathered}
$$

By differentiation and some algebra, we obtain

$$
\left.\begin{array}{rl}
f^{(t)}(x) & =\pi^{(t)} \cdot\left(e^{x} \cdot \boldsymbol{I}_{(0,1)}(x)+\left(1-e^{-1} x\right) e^{x} \cdot \boldsymbol{I}_{(1, t)}(x)\right), \\
\pi^{(t)} & =\frac{1}{e^{t-1}+e^{t}-e^{t-1} t} .
\end{array}\right\}
$$


From (3.15), (3.16), and Remark 3.2,

$$
M(t)=e^{t-1}+e^{t}-e^{t-1} t-1, \quad E\left(N_{t}\right)=e^{t-1}+e^{t}-e^{t-1} t .
$$

(Note that $\left.\lim _{t \uparrow 2} \pi^{(t)}=1 /\left(-e+e^{2}\right)\right), \quad M(2)=\left(-e+e^{2}-1\right), \quad E\left(N_{2}\right)=-e+e^{2}$. Also, $\lim _{t \downarrow 1} \pi^{(t)}=e^{-1}, M(1)=e-1, E\left(N_{1}\right)=e$, which agrees with Ross [22]).

$\boldsymbol{f}_{\gamma_{t}}(\boldsymbol{x}) \quad$ Applying (4.7) and substituting from (5.13) gives

$$
\begin{aligned}
f_{\gamma_{t}}(x) & =\int_{y=t+x-1}^{1} \frac{f_{0}^{(t)}(y)}{\pi^{(t)}} d y+\int_{y=1}^{t} \frac{f_{1}^{(t)}(y)}{\pi^{(t)}} d y=\int_{y=t+x-1}^{1} e^{y} d y+\int_{y=1}^{t}\left(1-e^{-1} y\right) e^{y} d y \\
& =-e^{t+x-1}+e^{t-1}+e^{t}-e^{t-1} t, \quad 0<x<(2-t), \\
f_{\gamma_{t}}(x) & =\int_{t+x-1}^{t} \frac{f_{1}^{(t)}(y)}{\pi^{(t)}} d y=\int_{y=t+x-1}^{t}\left(1-e^{-1} y\right) e^{y} d y \\
& =-e^{t+x-1}-2 e^{t+x-2}+e^{t+x-2} t+e^{t+x-2} x+e^{t}+e^{t-1}-e^{t-1} t, \quad(2-t)<x<1 .
\end{aligned}
$$

If $t \downarrow 1$ then $f_{\gamma_{t}}(x)=e-e^{x}, x \in(0,1)$. If $t=2$, then $f_{\gamma_{t}}(x)=-e^{1+x}+e^{x} x+e^{2}-e, x \in$ $(0,1)$. Also, $E\left(\gamma_{t}\right)=\frac{1}{2} e^{t-1}+\frac{1}{2} e^{t}-\frac{1}{2} e^{t-1} t-t, t \in(1,2]$. It is straightforward to check that $\int_{0}^{1} f_{\gamma_{t}}(x) d x=1$.

$\left\{\boldsymbol{\pi}_{\delta}^{(t)}, \boldsymbol{f}_{\delta_{t}}(\boldsymbol{x})\right\} \quad$ Substituting from (5.13) into (4.12) gives

$$
f_{\delta_{t}}(x)= \begin{cases}(1-x) \cdot\left(1-e^{-1}(t-x)\right) \cdot e^{t-x}, & 0<x<(t-1) \\ (1-x) \cdot e^{t-x}, & (t-1)<x<1\end{cases}
$$

which has a jump discontinuity at $x=t-1$ of magnitude

$$
f_{\delta_{t}}\left((t-1)^{+}\right)-f_{\delta_{t}}\left((t-1)^{-}\right)=2-t, \quad t \in[1,2) .
$$

Also $\int_{0}^{1} f_{\delta_{t}}(x) d x=1, \pi_{\delta}^{(t)}=0$, and $E\left(\delta_{t}\right)=-e^{t}+e^{t-1} t-e^{t-1}+1+2 t$.

$\boldsymbol{f}_{\beta_{t}}(\boldsymbol{x}) \quad$ Applying (4.16) and (4.17)

$$
f_{\beta_{t}}(x)= \begin{cases}\int_{y=0}^{x}\left(1-e^{-1}(t-y)\right) e^{t-y} d y, & 0<x<(t-1), \\ \int_{y=0}^{t-1}\left(1-e^{-1}(t-y)\right) e^{t-y} d y+\int_{y=t-1}^{x} e^{t-y} d y, & (t-1)<x<1 .\end{cases}
$$

Thus,

$$
f_{\beta_{t}}(x)= \begin{cases}e^{t}-e^{t-1} t+e^{t-1}-e^{t-x}+e^{t-x-1} t-e^{t-x-1}-e^{t-x-1} x, & 0<x<(t-1), \\ e^{t}-e^{t-1} t+e^{t-1}-e^{t-x}, & (t-1)<x<1,\end{cases}
$$

which is continuous at $x=(t-1)$ since

$$
\lim _{x \downarrow(t-1)} f_{\beta_{t}}(x)=\lim _{x \uparrow(t-1)} f_{\beta_{t}}(x)=e^{t}-e^{t-1} t+e^{t-1}-e .
$$

In addition, $E\left(\beta_{t}\right)=\frac{1}{2} e^{t-1} t-\frac{1}{2} e^{t}-\frac{1}{2} e^{t-1}+t+1$. 
Limiting proportion of renewals of $\left\{\boldsymbol{Z}_{n}\right\}_{n=1,2, \ldots}$ in $(\boldsymbol{r}, \boldsymbol{v}) \subseteq(\mathbf{0}, \boldsymbol{t})$. Suppose $0<r<1<$ $v<t$. From (5.11), and (5.13) we obtain

$$
p_{(r, v)}^{(t)}=\frac{-e^{r}+e^{v}+e^{(v-1)}-e^{(v-1)} v}{-1+e^{t}+e^{(t-1)}-e^{(t-1)} t} .
$$

For example, if $r=0.2, v=1.3, t=1.7$ then $p_{(r, v)}^{(t)}=0.666685$ (whereas $p_{(r, v)}^{(t)}=\frac{v-r}{t}=$ 0.6471 in (5.12) for exponential inter-arrival times.)

\subsection{Modified Renewal Process}

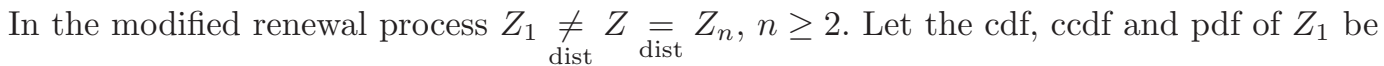
$B_{1}(\cdot), \bar{B}_{1}(\cdot)$, and $b_{1}(\cdot)$, respectively, with support $\left(0, U_{1}\right)$. The equilibrium renewal process is a modified renewal process where $b_{1}(x)=\frac{1}{E(Z)} \bar{B}(x)=\lim _{t \rightarrow \infty} f_{\gamma_{t}}(x)$ assuming $E(Z)$ exists (Cox [6], pp. 27-28).

Analogous to (3.5), an integral equation for $f^{(t)}(x)$ is

$$
\pi^{(t)} \bar{B}_{1}(x)+\int_{y=0}^{x} \bar{B}(x-y) f^{(t)}(y) d y=\pi^{(t)}, \quad 0<x<t
$$

Solving (5.19) with (3.6) yields $\left\{\pi^{(t)}, f^{(t)}(x)\right\}$.

Consider an example where $\bar{B}_{1}(x)=e^{-\mu_{1} x}, x>0$, and $\bar{B}(x)=e^{-\mu x}, x>0$; thus $U_{1}=$ $U=\infty$.

$\left\{\boldsymbol{\pi}^{(t)}, \boldsymbol{f}^{(t)}(\boldsymbol{x})\right\} \quad$ Substituting $\bar{B}_{1}(x)=e^{-\mu_{1} x}, \bar{B}(x)=e^{-\mu x}$ in (5.19), taking $d / d x$, and using (3.6) gives

$$
\begin{aligned}
f^{(t)}(x) & =\pi^{(t)}\left(\mu+\left(\mu_{1}-\mu\right) e^{-\mu_{1} x}\right), 0<x<t, \\
\pi^{(t)} & =\frac{1}{1+\mu t+\left(1-\frac{\mu}{\mu_{1}}\right)\left(1-e^{-\mu_{1} t}\right)} .
\end{aligned}
$$

$\boldsymbol{f}_{\gamma_{t}}(\boldsymbol{x}) \quad$ Substituting (5.21) into (4.4a) yields

$$
\begin{aligned}
f_{\gamma_{t}}(x) & =\mu_{1} e^{-\mu_{1}(t+x)}+\int_{y=0}^{t} \mu e^{-\mu(t+x-y)}\left(\mu+\left(\mu_{1}-\mu\right) e^{-\mu_{1} y}\right) d y, \\
& =\mu_{1} e^{-\mu_{1}(t+x)}+\mu e^{-\mu x}-\mu e^{-\left(\mu x+\mu_{1} t\right)}, \quad 0<x<\infty .
\end{aligned}
$$

$$
\begin{aligned}
\boldsymbol{f}_{\delta_{t}}(\boldsymbol{x}) \quad \text { By }(4.11) \\
\pi_{\delta_{t}}=e^{-\mu_{1} t}, \quad f_{\delta_{t}}(x)=e^{-\mu x}\left(\mu+\left(\mu_{1}-\mu\right) e^{-\mu_{1}(t-x)}\right), \quad 0<x<t .
\end{aligned}
$$


$f_{\beta_{t}} \quad$ From (4.13) and (4.14)

$$
\begin{aligned}
f_{\beta_{t}}(x)= & \left(\mu e^{-\mu x} \int_{y=0}^{x}\left(\mu+\left(\mu_{1}-\mu\right) e^{-\mu_{1}(t-y)}\right) d y\right) \boldsymbol{I}_{(\mathbf{0}, \mathbf{t})}(x) \\
& +\left(\mu_{1} e^{-\mu_{1} x}+\mu e^{-\mu x} \int_{y=0}^{t}\left(\mu+\left(\mu_{1}-\mu\right) e^{-\mu_{1}(t-y)}\right) d y\right) \boldsymbol{I}_{(\mathbf{t}, \infty)}(x), \\
f_{\beta_{t}}(x)= & \begin{cases}\frac{\mu e^{(-\mu x)}\left(-e^{-\mu_{1} t} \mu_{1}+e^{-\mu_{1} t} \mu+\mu x \mu_{1}+e^{-\mu_{1}(t-x)} \mu_{1}-e^{-\mu_{1}(t-x)} \mu\right)}{\mu_{1}}, & 0<x<t, \\
\mu_{1} e^{-\mu_{1} x}+\frac{\mu e^{-\mu x}\left(-e^{-\mu_{1} t} \mu_{1}+e^{-\mu_{1} t} \mu+\mu \mu_{1} t+\mu_{1}-\mu\right)}{\mu_{1}}, & t<x<\infty .\end{cases}
\end{aligned}
$$

It is readily shown that $\int_{0}^{\infty} f_{\beta_{t}}(x) d x=1$.

Note that $f_{\beta_{t}}(x)$ has a jump discontinuity at $x=t$ of magnitude $f_{\beta_{t}}\left(t^{+}\right)-f_{\beta_{t}}\left(t^{-}\right)=$ $\mu_{1} e^{-\mu_{1} t}$.

\section{CONCLUSIONS}

Many results in this paper can be derived by the conventional renewal integral equation approach, and some have been noted (e.g., formulas (3.10)-(3.12), (5.9)). The agreement of results tends to confirm the validity of the new analysis. There are important differences between the two methods, however. The new analysis is easier and faster if $t>U$ (Sections 4 and 5.2); and is straightforward and useful if $t \leq U$ (Section 4). The new approach shows immediately that the time- $t$ quantities exist for no-mean inter-arrival times, because of the existence of the pdf $\left\{\pi^{(t)}, f^{(t)}(x)\right\}_{0<x<t}$ (Section 2). It obtains the following quantities, not generally discussed explicitly in the renewal-theory literature: the time- $t$ quantities in modified renewal processes (Section 5.3); the pdf of $X_{J B}^{(t)}$ (Section 4.2); a result on the proportion of renewals in subsets of the time interval $(0, t)$ (Section 3.3). The new method routinely identifies discontinuities in the time- $t$ pdf's, not usually stressed in the literature (Sections 4.1.1, 4.3.1, 4.4.1, 5.1, 5.2, and 5.3). An important contribution of the new analysis is that it connects the time- $t$ renewal quantities with quantities in stochastic models where the limiting pdf of the state variable is fundamental to the solution, and there is a significant stopping time in the model (Section 1). Another contribution is that it gives a novel perspective of the time- $t$ quantities, which may be useful for students, researchers and practitioners. The new analysis is a useful addition to the methods now available for analyzing the finite time-t quantities of renewal theory (Sections 1, 2 and 3.1).

\section{Acknowledgments}

The author thanks the referee for insightful comments and suggestions, which have helped to improve the paper. This work was supported by the Natural Sciences and Engineering Research Council of Canada.

\section{References}

1. Feller, W. (1941). On the integral equation of renewal theory. Annals of Mathematical Statistics 12(3): 243-267.

2. Feller, W. (1950). An introduction to probability theory and its applications, Volume I, New York: John Wiley

3. Feller, W. (1971). An introduction to probability theory and its applications, Volume II, 2nd edn., New York: Wiley. 
4. Doob, J.L. (1948). Renewal theory from the point of view of the theory of probability. Transactions of the American Mathematics Society 63(3): 422-438.

5. Smith, W.L. (1958). Renewal theory and its ramifications. Journal of the Royal Statistical Society, Series B (Methodological), 20(2): 243-302.

6. Cox, D.R. (1970). Renewal theory (reprinted from 1962 edition), London: Methuen.

7. Ross, S.M. (1970). Applied probability with optimization applications, San Francisco: Holden Day.

8. Karlin, S. \& Taylor, H.M. (1975). A first course in stochastic processes, 2nd edn., New York: Academic Press.

9. Bartholemew, D.J. (1963). An approximate solution of the integral equation of renewal theory. Journal of the Royal Statistical Society, Series B (Methodological) 25(2): 432-441.

10. Deligonul, S.S. (1985). An approximate solution of the integral equation of renewal theory. Journal of Applied Probability 22: 926-931.

11. Sigman, K. \& Wolff, R.W. (1993). A review of regenerative processes, SIAM Review 35(2): 269-288.

12. Asmussen, S. (2003). Applied probability and queues. New York: Springer-Verlag.

13. Tijms, H.C. (2003). A first course in Stochastic models, New York: Wiley

14. Brill, P.H. (2009). Compound cycle of a renewal process and applications. Information Systems and Operational Research 47(4): 273-281.

15. Brill, P.H. \& Yu, K. (2011). Analysis of risk models using a level crossing technique. Insurance: Mathematics and Economics 49(3): 298-309.

16. Brill, P.H. \& Posner, M.J.M. (1977). Level crossings in point processes applied to queues: single-service case. Operations Research 25(4): 662-674.

17. Brill, P.H. \& Posner, M.J.M. (1981). The system point method in exponential queues: a level crossing approach. Mathematics of Operations Research 6: 31-49.

18. Cohen, J.W. (1976). On Regenerative Processes in Queueing Theory. Lecture Notes in Economics and Mathematical Systems. (M. Beckman and H.P. Kunzi Eds.), New York: Spring-Verlag.

19. Cohen, J.W. (1977). On up- and down-crossings. Journal of Applied Probability 14: 405-410.

20. Brill, P.H. (2000). A brief outline of the level crossing method in Stochastic models, CORS Bulletin Canadian Operational Research Society 34(4): 9-21. (http://www.cors.ca/bulletin/percy.pdf).

21. Brill, P.H. (2008). Level crossing methods in Stochastic models. New York: Springer.

22. Ross, S.M. (2006). Introduction to probability models, 9th edn., San Diego: Academic Press.

23. Pinsky M. \& Karlin, S. (2011). Introduction to Stochastic modelling, 4th edn., San Diego: Academic Press.

24. Wolff, R.W. (1982). Poisson arrivals see time averages, Operations Research 30(2): 223-231.

25. Gerber, H.U. \& Shiu, E.S.W. (1997). The joint distribution of the time of ruin, the surplus immediately before ruin, and the deficit at ruin. Insurance: Mathematics and Economics 21: 129-137.

26. Botta, R.F., Marchal, W.D. \& Harris, C.M. (1987). Characterizations of generalized hyperexponential distribution functions. Stochastic Models 3: 115-148.

27. Botta, R.F. \& Harris, C.M. (1986). Approximation with generalized hyperexponential distributions. Weak convergence results. Queueing Systems 2: 169-190.

28. Harris, C.M., Marchal, W.G. \& Botta, R.F. (1992). A note on generalized hyperexponential distributions. Stochastic Models 8: 179-191.

29. Yu, K., Huang, M.L. \& Brill, P.H. (2012). An algorithm for fitting heavy-tailed distributions via generalized hyperexponentials. INFORMS J. on Computing 24: 42-52. 